\title{
Gravity vs. Dark Energy, about the Expansion of the Universe
}

\author{
Olivier Serret \\ ESIM Engineer, Cugnaux, France \\ Email: o.serret@free.fr
}

How to cite this paper: Serret, O. (2018) Gravity vs. Dark Energy, about the Expansion of the Universe. Journal of Modern Physics, 9, 84-97.

https://doi.org/10.4236/jmp.2018.91006

Received: December 22, 2017

Accepted: January 15, 2018

Published: January 18, 2018

Copyright $\odot 2018$ by author and Scientific Research Publishing Inc. This work is licensed under the Creative Commons Attribution International License (CC BY 4.0).

http://creativecommons.org/licenses/by/4.0/

\begin{abstract}
Dark energy is argued by the accelerating expansion of the Universe but has not been directly measured. In this article, some uncertainties are pointed out, first one being the determination of the Hubble constant. And the main parameters (magnitude, distance, redshift, velocity) are checked. Distinguishing the instantaneous velocity from the average velocity, it is then concluded from the same data that the expansion would not be accelerating, and that the Gravity would slightly slow down the motion of explosion. Moreover, at the end of the paper, it is proposed a neo-Newtonian approach to get the computed values in a closer agreement with the observed values; this Neo-Newtonian Mechanics is in coherence with the Quantum Mechanics.
\end{abstract}

\section{Keywords}

Acceleration, Expansion, Inflation, Universe, Cosmos, Magnitude, Redshift, Hubble Constant, Uncertainties of Measure, Dark Energy, Gravitation, Relativity, Neo-Newtonian Mechanics

\section{Introduction}

In 2011, the Nobel Prize in Physics was awarded "for the discovery of the accelerating expansion of the Universe through observations of distant supernovae". It rewards both difficult observations and mainly a clever explanation. The trouble comes from that a Nobel Prize is understood not only as a justified reward but also as a proof or an irremovable truth. Then the accelerating expansion is today a dogma which should not be criticized or even seriously discussed with the partisans of the theory of Relativity, despite their own inconsistencies: if the theory of Relativity can explain gravitational effect (by distortion of space-time), it cannot explain the "Standard Model" of Quantum Mechanics with the (other) three forces. And although a large majority (69\%) of the Universe would content 
it, in fact the dark energy has never been measured. It looks like a mysterious form of energy, and a few people begin to wonder about its reality [1]. It is why the purpose of this paper is, starting again from the observations of distant supernovae, to discuss about this explanation.

\section{Observation of Distant Supernovae}

A supernova is a sudden appearance of star (due to its explosion) before slowly fading from sight over several weeks or months. Supernovae of type I are considered to supply the same brightness and so are considered as candles whose distances can be computed. Cepheids too are used to estimate distances due to their relation between pulsation period and luminosity.

The redshift is the increase of the wavelength of light emitted from most of the stars. It is linked to the radial velocity.

Then it can be built a diagram to show the link between the magnitude (or the brightness) and the redshift. This link was initially established by Lemaittre and Hubble, and recently completed by Pr. Perlmutter [2], Schmidt and Riess with distant supernovae (see Figure 1).

"The high-redshift SNeIa from both teams are 0.28 mag dimmer or $14 \%$ farther than expected in a Universe with this much matter and no cosmological constant. The statistical uncertainty of these values is 0.08 and 0.06 mag (or 4\% and $3 \%$ in distance)" [3].

That means that the distance would be farther from $18 \%$ to only $10 \%$. Where could these uncertainties come from?

\section{Usual Explanations}

\subsection{The Hubble Constant}

Edwin Hubble [4] found the relation between the velocity and the distance to be:

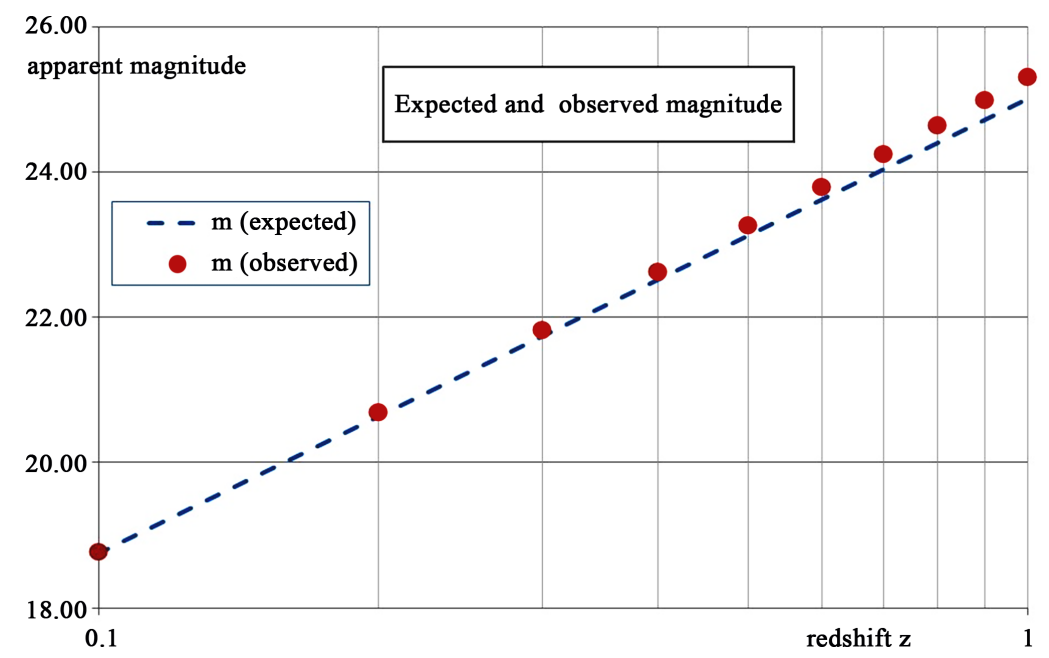

Figure 1. Magnitude according to redshift. Observed data extracted from "Supernovae, Dark Energy, and the Accelerating Universe" [2], Figure 3 Expected values in dashed line with $H o=70 \mathrm{~km} / \mathrm{s} / \mathrm{Mpc}$, Formula (B5-c) in Appendix B. 


$$
V=H o \cdot D
$$

with $\mathrm{Ho}$ being a Constant which carries his name and which value he found was close to

$$
H o \approx 500 \mathrm{~km} / \mathrm{s} / \mathrm{Mpc}
$$

Today, the value of this constant is considered to be between 50 and 80 , close to 67 or 72 (see Figure 2), which is $7 \%$ of uncertainty, the order of magnitude on distance difference.

How could Pr Hubble do such a mistake in his measurements? The measure of the redshift is very precise, and the relation between the redshift and the velocity has not changed. The difference comes from the measure of the brightness and the estimation of distance which, for a poorly explained reason, were absolutely wrong. A difference of magnitude of 6 is a difference of observed brightness of about 40 times (see Appendix A).

If the values of magnitudes and distances have been now corrected, however it does no match exactly with a Constant value. But the idea of the constant link between velocity and distance remains in the scholar community, and it is the meaning of the blue dashed lines in Figure 1.

\subsection{The Accelerating Expansion of the Universe}

Due to the Hubble "constant", it was then considered that the expansion of the Universe should be "constant" (i.e. along the dashed lines of Figure 1). With the progresses of the measuring means, it is now possible to observe supernovae more and more distant. And they do not follow the expected path. So, the explanation given by Perlmutteris: "In particular, you can think of making a measurement of a supernova explosion at one given time in history. If, for example, you found [less] redshift at that time than expected from the current expansion rate, that would imply that the expansion was [slower] in the past and has been [accelerating]. This would lead you to conclude that there was a [lower] mass density in the universe." [5].

Let us illustrate this argument by a graph in Figure 3.

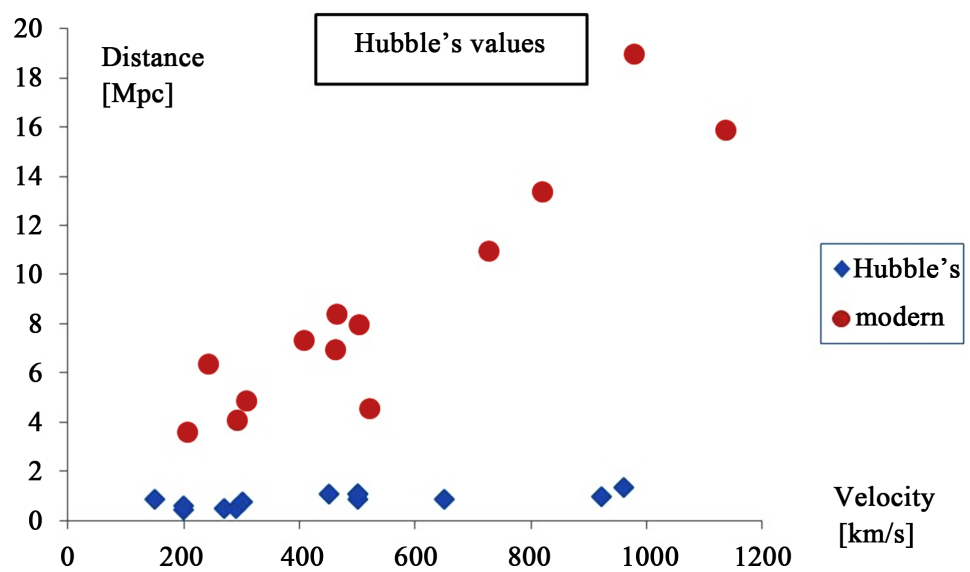

Figure 2. Differences between values found by Pr. Hubble and modern values.

${ }^{1}$ Terms in square brackets [] have been modified in order to get the direct explanation. 


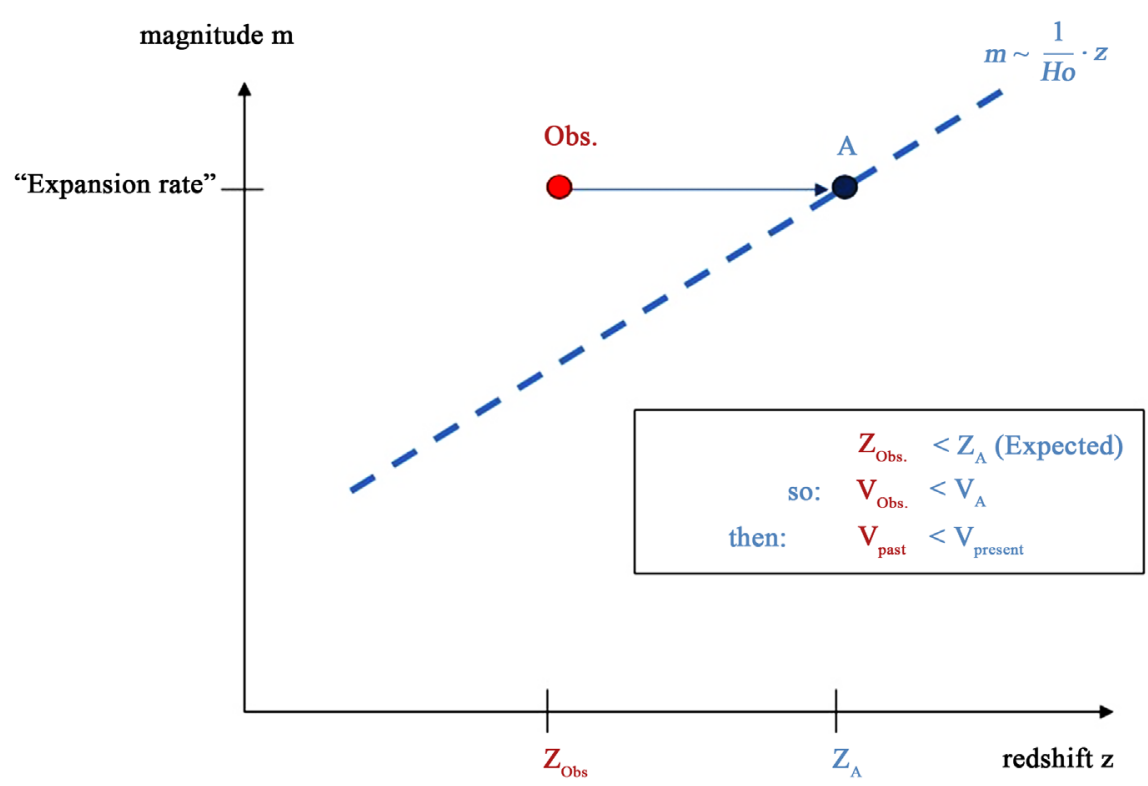

Figure 3. Graph of the expansion increasing, a horizontal comparison.

May we discuss the fuzzy assertion about the "expected" redshift in the past with the consequence that expansion would have been slower in the past? Can another analysis be proposed?

\section{Some Alternative Explanations}

\subsection{Absorption}

Some diagrams are given in "observed" magnitude [2], which would mean "strictly observed, without correction". If we had some absorption due to the dust in vacuum, it could decrease the brightness of the star, and so increase its apparent magnitude. For example, to correlate the data with the Hubble law, we took dust absorption of 1.7; an increase of length of $14 \%$ is equivalent to increase dust absorption of 0.3, i.e. to get a magnitude of 24.3 (instead of 24.0).

Of course we are confident that such a correction is already done in observed magnitude (see Appendix B). But because the value of absorption on such a distance cannot be precisely measured and could be so huge (see Appendix C) and because the hypotheses taken on the absorption coefficient are not given in the articles, this trivial explanation is given "for memory".

\subsection{The Hubble Values}

It is difficult to determine its value. For Pr. Edwin Hubble, it was about 500. With the measures of Hubble telescope on Cepheids, it is $72 \mathrm{~km} / \mathrm{s} / \mathrm{Mpc}$ but with the measures of Plank satellite on far supernovae, it is $67 \mathrm{~km} / \mathrm{s} / \mathrm{Mpc}$. We can note that Cepheids are closer to Earth than Supernovae; maybe is there a link between the computed Hubble value and the distance. Then it would be conclude that the Hubble constant decreases with the distance, which would mean that the Hubble constant is not a constant! 
Another way to express the Hubble law is:

$$
D=V \cdot T o
$$

with

$$
T o=1 / H o
$$

For example, for $H o=70 \mathrm{~km} / \mathrm{s} / \mathrm{Mpc}, T o=\left(3.085 \times 10^{19} / 70=4.4 \times 10^{17} \mathrm{~s} / 3.15\right.$ $\left.\times 10^{7}=\right) 14$ billions of years. If the Hubble constant were not the same for distant and for close galaxies, that would mean the time since the Big Bang would be different either we are far or close from the Earth, which would be strange.

Let us check another hypothesis. The photons of the far galaxies were emitted a long time ago. Let us call it this moment $T^{\prime}$, with $T^{\prime}<T o$. Then by definition of $H^{\prime}$ (see Table 1 ), $H^{\prime}>H o$, and so for a same $z$ (or velocity), the observed distance $D^{\prime}$ would look smaller than expected. But it is the contrary it has been measured, a greater distance for the same $z$. How can we explain this paradox?

\subsection{The Big Bang Origin}

With the Hubble law and the redshift, quite all the galaxies seem to move away from each other. It is usually given the picture of a pancake in an oven where all the grapes move away from each other. In the theory of Relativity, there is no centre. We can check in Appendix D that we can explain it within the Newton Mechanics, with the single difference that there would be a centre for the Big Bang.

Then the Relativity theory is not the single explanation for the Big Bang and expansion of the universe can be seen from another point of view, with a centre.

\subsection{The Observed Hubble Values}

Let us now say that all the observations of redshift $Z$ and magnitude $M$ are correct, that the computing of velocity $V$ and distance $D$ are correct too, and that the Earth is not so far from the centre.

And let us suppose that at the Big Bang origin, all the galaxies were ejected at different velocities (like in a bomb). The speeder one will go farther. In a sphere,

\begin{tabular}{|c|c|c|}
\hline Symbol & Property & Definition \\
\hline Ho & & Hubble constant(an average of calculated values) \\
\hline To & $1 / H o$ & Present time or duration from the origin \\
\hline$T^{\prime}$ & $T^{\prime}<T o$ & Time of the observed event \\
\hline$H^{\prime}$ & $1 / T^{\prime}$ & H value calculated from time $T^{\prime}$ at a specific point \\
\hline$D^{\prime}$ & $D(T)$ & Distance at the time $T^{\prime}$ \\
\hline $\boldsymbol{V}^{\prime}$ & $v(T)$ & Observed velocity at the time $T^{\prime}$ \\
\hline$v_{a v}^{\prime \prime}$ & $D^{\prime} / T^{\prime}$ & Average velocity from the origin to the time $T^{\prime}$ \\
\hline$H(o b s)$ & $v^{\prime} / D^{\prime}$ & $\begin{array}{l}\text { Observed } \mathrm{H} \text { value, or } \mathrm{H} \text { value calculated from the } \\
\text { velocity and the distance at a specific point. }\end{array}$ \\
\hline
\end{tabular}

Table 1. Definitions of symbols. 
the value of gravity is in correlation with the radius of the sphere, with the volume more precisely. Then the influence of gravity will be higher for distant galaxies than for close galaxies (from the centre).

In fact, the more the star or galaxy is fast, the more the distance is important, and so the older the event is.

Let us have the definitions hereafter (see Table 1).

The path the photon used from a distant event is:

$$
\begin{gathered}
D\left(T^{\prime}\right)=c\left(T o-T^{\prime}\right) \\
D\left(T^{\prime}\right)=v^{\prime \prime} T^{\prime}
\end{gathered}
$$

So

$$
H^{\prime}=\left(1+v^{\prime \prime} / c\right) H o
$$

Because velocity is linked to $Z$, It confirms that $H^{\prime}$ (see the dashline in Figure 2) should slightly increase with $z$ (and so the dashed curve should slightly decrease):

Note: it could give the impression that the observed universe is more increasing than previously

Because observed points are above the dashed curve, we can conclude that

$$
H(o b s)<H^{\prime}
$$

\subsection{The Gravity}

By definition of $H(o b s)$ :

$$
D^{\prime} \cdot H(o b s)=v^{\prime}
$$

And by definition of the time $T^{\prime}$ when occurred the event:

$$
D^{\prime} \cdot H^{\prime}=v^{\prime \prime}(a v)
$$

Then

$$
D^{\prime}=v^{\prime \prime}(a v .) / H^{\prime}=v^{\prime} / H(o b s)
$$

Because $H(o b s)$ is lower than $H^{\prime}$ (cf. Equation (8)), then

$$
v^{\prime}<v^{\prime \prime}(a v)
$$

Velocity at the event time $T^{\prime}$ is lower than the average velocity, so the initial velocity was greater. Then we can conclude that the expansion is not accelerating, and it is even decreasing from our point of view.

Then the velocity will slightly decrease with the time (see Figure 4).

It would mean the gravity slow down the expansion, and that the formula would look like:

$$
D^{\prime}=\int_{T_{\text {initial }}}^{T^{\prime}}\left(V_{\text {initial }}-\frac{G \cdot M \cdot t}{D(t)^{2}}\right) \mathrm{d} t
$$

To sum up the purpose, let us check Figure 5. 


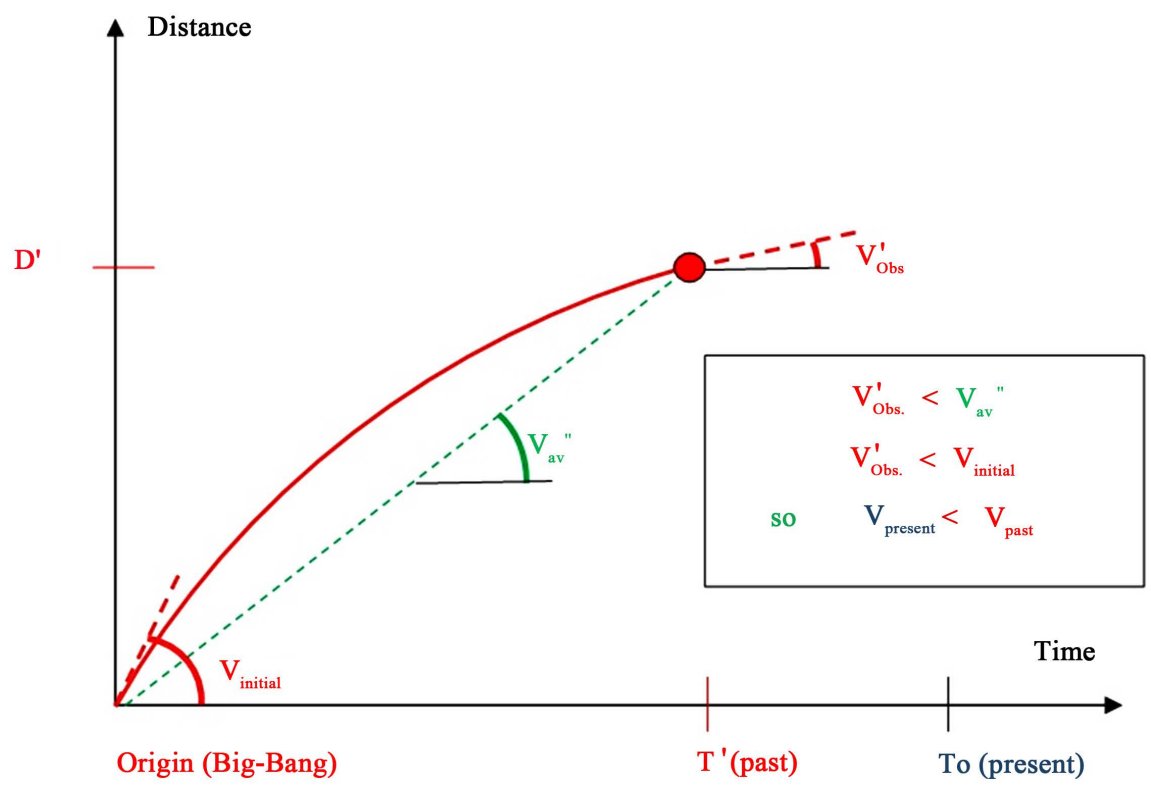

Figure 4. Distance in function of time.

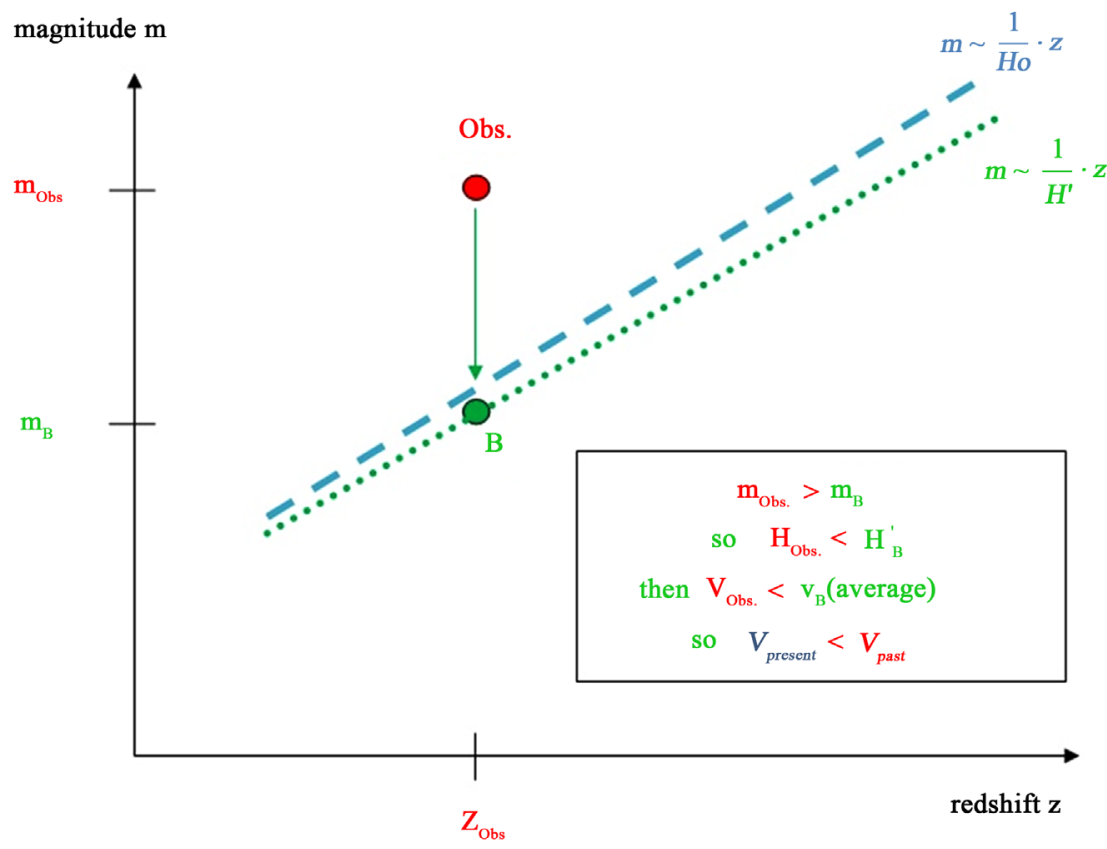

Figure 5. An alternative explanation, a vertical comparison.

\subsection{The Redshift}

The other axis of Figure 1 is the redshift. Can we get another relation between the redshift and the velocity? According to the relativity theory, it is the Equation hereafter:

$$
\frac{v}{c}=\frac{(1+z)^{2}-1}{(1+z)^{2}+1}
$$

According to the Newtonian mechanics, it is: 


$$
1+z=\frac{1+v_{\text {source }} / c}{1+v_{\text {obs }} / c}
$$

The limit with the Newtonian mechanics is it does not work at very high speed, especially with the addition of velocities. But if we apply the Doppler effect of the Newtonian Mechanics, and assuming that the Sun (the observer) would be motionless (it is a simplification):

$$
z \approx \frac{v_{\text {source }}}{c}
$$

and so for a same $z$, the value of velocity is higher with neo-Newtonian mechanics than with Relativity. Then the distance computed with the Hubble law would be higher with neo-Newtonian mechanics (see Appendix E) than with Relativity theory (see Figure 6). This would mean the velocity of expansion is decreasing (due to the internal Gravity) but is quasi-constant.

\section{Conclusion}

Dark energy is argued by the acceleration of the expansion of Universe but has never been directly measured. When we check the data, this explanation is based on the difference between the observed magnitude and the computed magnitude for a given redshift. But there are a lot of uncertainties; the first one is about the Hubble constant; the second one is on the "expected" redshift. Then it has been checked in this article the influence of the dust absorption, and the distinctions between the Hubble values. Establishing the distinction between the instantaneous

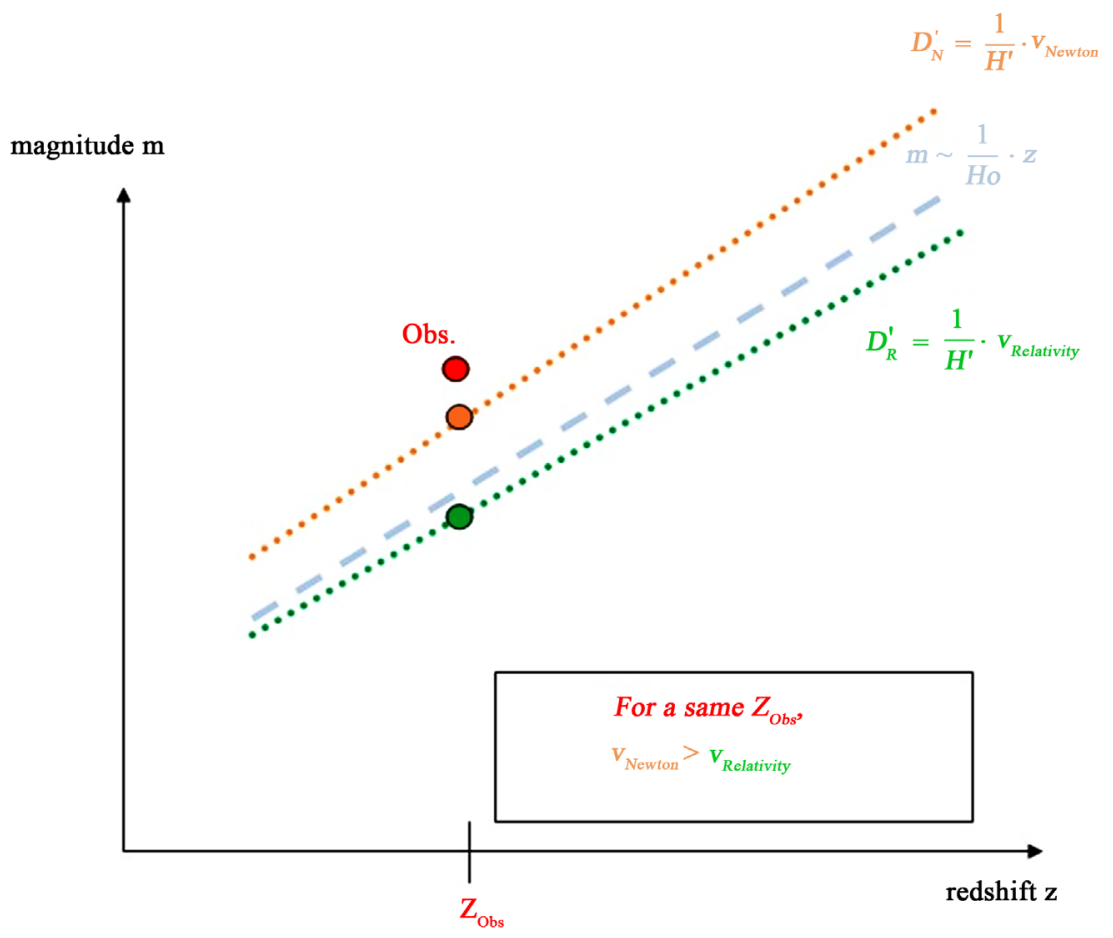

Figure 6. Relations between redshift and velocities (Relativity velocity and Newtonian velocity). 
velocity and the average velocity, from the same data we can conclude at the opposite that the expansion is slightly slowing down due to the Gravity! In addition, using the neo-Newtonian mechanics, the calculated magnitude would be very close to the observed magnitude; let us recall that the Neo-Newtonian Mechanics is coherent with the Quantum Mechanics.

\section{References}

[1] Cho, A. (2017) Is Dark Energy an Illusion? Science. http://www.sciencemag.org/news/2017/04/dark-energy-illusion

[2] Perlmutter, S. (2003) Supernovae, Dark Energy, and the Acceleration Universe. Physics Today, 53-60. http://www-supernova.lbl.gov/PhysicsTodayArticle.pdf

[3] Riess A. (2000) The Case for an Accelerating Universe from Supernovae. PASP, 1-33. https://arxiv.org/pdf/astro-ph/0005229.pdf

[4] Hubble, E. (1929) Proceedings of the National Academy of Sciences, 15, No. 3. https://apod.nasa.gov/diamond_jubilee/d_1996/hub_1929.html

[5] Perlmutter, S. (1999) Supernovae, Dark Energy, and the Accelerating Universe: The Status of the Cosmological Parameters. Stanford Symposium, 733-756. http://www.slac.stanford.edu/econf/C990809/docs/perlmutter.pdf

[6] Duriez, L. (2003) Instruments Astronomiques. USTL, p. 8. http://lal.univ-lille1.fr/Astro_DeugSM2.pdf

[7] Scannapieco, E. (2007) Nuages et vides intergalactiques. Pour la Science. http://www.pourlascience.fr/ewb_pages/a/article-nuages-et-vides-intergalactiques-2 0243.php 


\section{Appendix A: Brightness}

Formula of Brightness $F$ :

$$
F=\frac{L}{4 \Pi D^{2}}
$$

with $L$ the Luminosity $\left(=6 \times 10^{19}\right.$ SI for SN $)$

Formula of apparent magnitude $\mathrm{m}$ :

$$
D=10^{\frac{m-M-A+5}{5}}
$$

With $M$, absolute magnitude (=-19.3 for SN Ia)

According to the Hubble law:

$$
D \cdot H o=v
$$

With $H o \approx 70 \mathrm{~km} / \mathrm{s} / \mathrm{Mpc}$

And according to the Relativity theory:

$$
\frac{v}{c}=\frac{(1+z)^{2}-1}{(1+z)^{2}+1}
$$

Then, it can be built Figure A1 hereafter. A variation of magnitude of 6 is 40 times a variation of brightness.

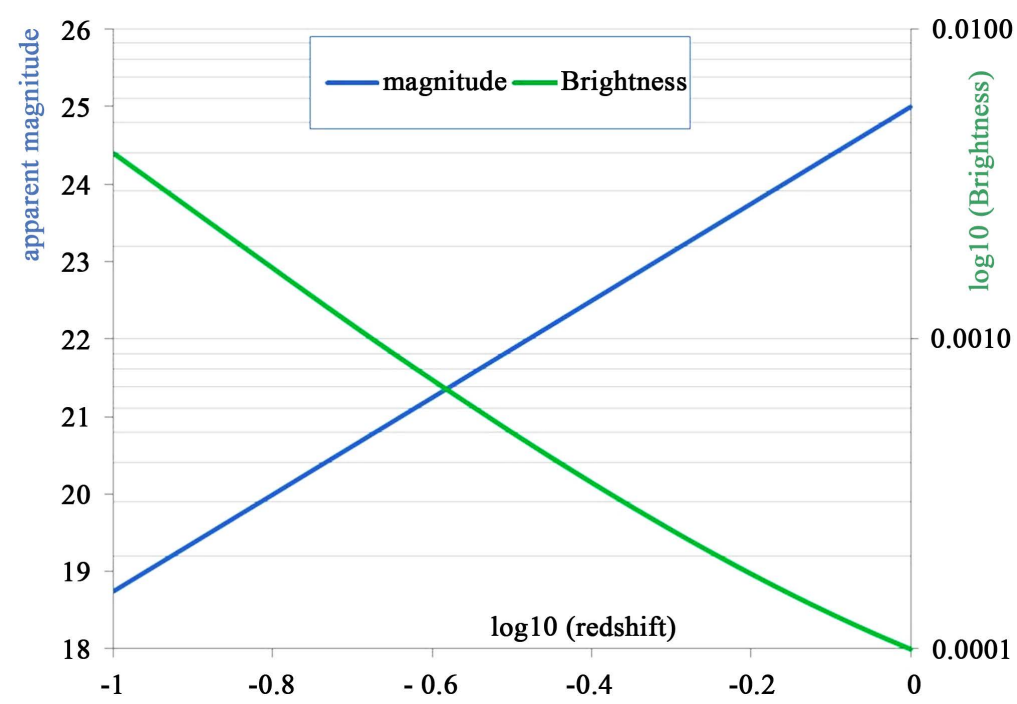

Figure A1. Magnitude vs. brightness.

\section{Appendix B: Absorption and Magnitude}

1) According to observations,

For $z=0.7, \underline{m=24}$

2) According to Hubble law:

$$
D \cdot H o=V
$$

And according to the Relativity theory:

$$
\frac{v}{c}=\frac{(1+z)^{2}-1}{(1+z)^{2}+1}
$$


And according to the formula of apparent magnitude:

$$
D=10^{\frac{m-M-A+5}{5}} \cdot 10^{-6}
$$

with $M=-19.3$ for SN la, and $D$ in $[\mathrm{Mpc}]$

Then:

$$
V=10^{\frac{m-M-A+5}{5}} 10^{-6} \cdot H o=c \cdot \frac{(1+z)^{2}-1}{(1+z)^{2}+1}
$$

or

$$
m=5 \cdot\left(\log _{10}\left(\frac{c}{H o} \cdot \frac{(1+z)^{2}-1}{(1+z)^{2}+1}\right)+6\right)+M+A-5
$$

With the same example, for $z=0.7$ (and $A=0$ ), we get $\underline{\boldsymbol{m}=22.3}$.

3) To get a coherent value with the observation, we have to add a dust absorption of $A=1.74$.

Then for $z=0.7$ and $A=1.74$, we get $\underline{m=24}$.

\section{Appendix C: Vacuum Absorption}

On Earth, the air absorption is about $0.01 / \mathrm{km}$ at the atmospheric pressure [6]; on a length of $8 \mathrm{~km}$, Absorption $A=0.08$ and Transmission $T=10^{-0.08}$. For air at atmospheric pressure, there are $6.023 \times 10^{23}$ molecules per 22.4 Liters, or $3 \times 10^{25}$ molecule $/ \mathrm{m}^{3}$. In vacuum, the intergalactic space is expected to get 10 atoms $/ \mathrm{m}^{3}$ [7]. When we look to one direction, the photon crosses a space with $10^{8}$ less atoms, then absorption would be of about $10^{-10} / \mathrm{km}$. On a distance of $200 \mathrm{Mps}$, or $10^{20} \mathrm{~km}$, it could be an absorption of $10^{10}$ or a transmission of $10^{\wedge}-\left(10^{\wedge} 10\right)$, which means nothing!

Air:

Molecules: $6 \times 10^{23} / 22.4 \mathrm{~L}=3 \times 10^{25} / \mathrm{m}^{3}$

$k($ air $)=0.01 / \mathrm{km}$

$$
A=k \cdot D=0.01 \times 8=0.08
$$

Transmission $=T=10^{-0.08}$

With $A \approx 0$, then $T \approx 1$

There is a good transmission

Vacuum:

10 atoms $/ \mathrm{m}^{3}=2$ atom/direction

$30 \times 10^{24}$ molecule $/ \mathrm{m}^{3}=3 \times 10^{8}$ molecule/direction

$10^{8}$ less in vacuum than in space

$K^{\prime}($ vacuum $)=10^{-10} / \mathrm{km}$

$200 \mathrm{Mpc}=200 \times 3 \times 10^{19} \mathrm{~km}=6 \times 10^{21} \mathrm{~km}$

Absorption $A^{\prime}=10^{-10} \times 6 \times 10^{21}=6 \times 10^{11}=$ infinit

Transmission $T^{\prime}=10^{- \text {infinite }}$

With $A \approx$ infinite, then $T \approx 0$

On such a huge value, it could have a poor transmission. 


\section{Appendix D: Newtonian Big Bang}

Let us suppose Velocity $V$ is proportional to the Distance $D$ (as defined by the Hubble law) in the case of an explosion:

$$
V=D \cdot H
$$

And we consider we are not at relativistic velocities (not close to the celerity c) and so we can add or subtract according to the usual Newtonian law.

\section{Coordinate of Earth:}

In the $\mathrm{O}$ frame, the Earth is at a distance of $2 D$, and then its velocity is $2 D$

In the $\mathrm{E}$ frame, the Earth is in the center of its own frame, so its distance is nil and its velocity too.

Coordinate of star A:

In the $\mathrm{O}$ frame, the star $\mathrm{A}$ is at a distance of $3 \mathrm{D}$, and then its velocity is $3 \mathrm{~V}$

In the $\mathrm{E}$ frame, the star $\mathrm{A}$ is at a distance of $(3 D-2 D=) 1 D$.

Its velocity is $(3 V-2 V=) 1 V$

Coordinate of star B:

In the $\mathrm{O}$ frame, the star $\mathrm{B}$ is at a distance of $1 D$, and then its velocity is $1 \mathrm{~V}$

In the $\mathrm{E}$ frame, the star $\mathrm{B}$ is at a distance of $(1 D-2 D=)-1 D$.

Its velocity is $(1 V-2 V=)-1 V$

Coordinate of star C:

In the $\mathrm{O}$ frame, the star $\mathrm{C}$ is at a distance of $-1 D \vec{i}$, and then its velocity is $-1 V \vec{i}$

In the $\mathrm{E}$ frame, the star $\mathrm{C}$ is at a distance of $(-1 D-2 D=)-3 D \vec{i}$.

Its velocity is $(-1 V-2 V=)-3 V$

Coordinate of star D:

In the $\mathrm{O}$ frame, the star $D$ is at a distance of $2 D \vec{j}$, and then its velocity is $2 D \vec{j}$

In the $\mathrm{E}$ frame, the star $D$ is at a distance of $\left(\sqrt{(2 D)^{2}+(2 D)^{2}}=\right) 2.8 D$.

Its velocity is on the horizontal $(0-2 V=)-2 V \vec{i}$ and on the vertical $(2 V-0$

$=)+2 \overrightarrow{V j}$, so its velocity is $\left(\sqrt{(2 V)^{2}+(2 V)^{2}}=\right) 2.8 V$.

\section{Coordinate of star F:}

In the $\mathrm{O}$ frame, the star $\mathrm{F}$ is at a distance such as $3 D \cdot \cos \theta=2 D$, so $\theta=48^{\circ}$.

Then its velocity is $3 V$ and $3 V \cdot \cos \left(48^{\circ}\right) \vec{i}+3 V \cdot \sin \left(48^{\circ}\right) \vec{j}=2 V \vec{i}+2.2 \overrightarrow{V j}$

In the $\mathrm{E}$ frame, the star $\mathrm{F}$ is at a distance of $\left(3 D \cdot \sin \left(48^{\circ}\right)=\right) 2.2 D$.

Its velocity is on the horizontal $(2 \mathrm{~V}-2 \mathrm{~V}=) \quad 0 \mathrm{Vi}$ and on the vertical $(2.2 \mathrm{~V}-$ $0=)+2.2 \overrightarrow{V j}$, so its velocity is $2.2 \mathrm{~V}$.

Coordinate of star G:

In the $\mathrm{O}$ frame, the star $\mathrm{G}$ is at a distance $\sqrt{(2 D)^{2}+(1 D)^{2}}=2.23 D$ and $\sqrt{(2 D)^{2}+(1 D)^{2}} \cdot \cos \theta=2 D$, so $\theta=26^{\circ}$.

Then its velocity is $2.23 \mathrm{~V}$ and

$$
2.23 \mathrm{~V} \cdot \cos \left(26^{\circ}\right) \vec{i}+2.23 \mathrm{~V} \cdot \sin \left(26^{\circ}\right) \vec{j}=2 V \vec{i}+\overrightarrow{V j}
$$

In the $\mathrm{E}$ frame, the star $\mathrm{G}$ is at a distance of $1 D$.

Its velocity is on the horizontal $(2 V-2 V=) \quad 0 V \vec{i}$ and on the vertical $(1 V-0$ 
$=) 1 \overrightarrow{V j}$, so its velocity is $1 \mathrm{~V}$.

Coordinate of star $\mathrm{H}$ :

In the $\mathrm{O}$ frame, the star $\mathrm{H}$ is at a distance $3 D$ at an angle of $\theta=30^{\circ}$.

Then its velocity is $3 \mathrm{~V}$ and $3 \mathrm{~V} \cdot \cos \left(30^{\circ}\right) \vec{i}+3 \mathrm{~V} \cdot \sin \left(30^{\circ}\right) \vec{j}=2.6 V \vec{i}+1.5 \mathrm{Vj}$

In the $\mathrm{E}$ frame, the star $\mathrm{H}$ is at a distance of $\sqrt{(0.6 D)^{2}+(1.5 D)^{2}}=1.6 D$.

Its velocity is on the horizontal $(2.6 V-2 V=) 0.6 V \vec{i}$ and on the vertical $(1.5 \mathrm{~V}-0=) 1.5 \mathrm{Vj}$, so its velocity is $1.6 \mathrm{~V}$.

Coordinate of star I:

In the $\mathrm{O}$ frame, the star $\mathrm{I}$ is at a distance $4 D$ and $4 D \cdot \cos \theta=1 D$, so $\theta=14.5^{\circ}$.

Then its velocity is $4 V$ and $4 V \cdot \cos \left(14.5^{\circ}\right) \vec{i}+4 V \cdot \sin \left(14.5^{\circ}\right) \vec{j}=3.9 V \vec{i}+1 V \vec{j}$

In the $\mathrm{E}$ frame, the star $\mathrm{I}$ is at a distance of $\sqrt{(1.9 D)^{2}+(1 D)^{2}}=2.14 D$.

Its velocity is on the horizontal $(3.9 V-2 V=) 1.9 V \vec{i}$ and on the vertical ( $1 \mathrm{~V}$ $-0=) 1 V \vec{j}$, so its velocity is $2.14 \mathrm{~V}$.

Coordinate of star J:

In the $\mathrm{O}$ frame, the star $\mathrm{J}$ is at a distance $2 D$ and the angle is $\theta=135^{\circ}$.

Then its velocity is $2 V$ and $2 V \cdot \cos \left(135^{\circ}\right) \vec{i}+2 V \cdot \sin \left(135^{\circ}\right) \vec{j}=-1.4 V \vec{i}+1.4 V \vec{j}$

In the $\mathrm{E}$ frame, the star $\mathrm{J}$ is at a distance of $\sqrt{(3.4 D)^{2}+(1.4 D)^{2}}=3.67 D$.

Its velocity is on the horizontal $(-1.4 V-2 V=)-3.4 V \vec{i}$ and on the vertical $(1.4 \mathrm{~V}-0=) 1.4 \overrightarrow{V j}$, so its velocity is $3.67 \mathrm{~V}$.

And so on... see Table A1 and Figures A2.
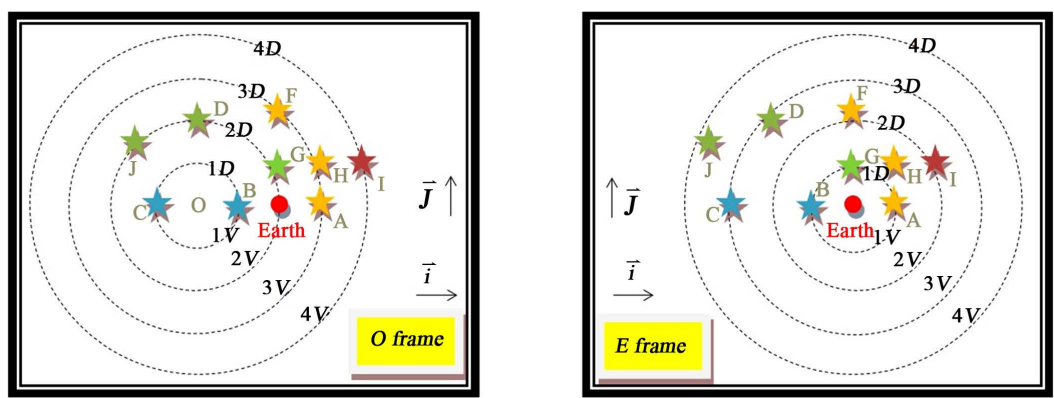

Figure A2. Distances and velocities in two reference frames.

Table A1. Distances and velocities in two reference frames.

\begin{tabular}{ccccc}
\hline \multirow{2}{*}{ STAR } & \multicolumn{2}{c}{ O frame } & \multicolumn{2}{c}{ E frame } \\
\cline { 2 - 5 } & Distance & Velocity & Distance & Velocity \\
\hline Earth & $2 D$ & $2 V$ & 0 & 0 \\
Star A & $3 D$ & $3 V$ & $1 D$ & $1 \mathrm{~V}$ \\
Star B & $1 D$ & $1 V$ & $-1 D$ & $-1 \mathrm{~V}$ \\
Star C & $-1 D$ & $-1 V$ & $-3 D$ & $-3 V$ \\
Star D & $2 D$ & $2 V$ & $2.8 D$ & $2.8 V$ \\
Star F & $3 D$ & $3 V$ & $2.2 D$ & $2.2 \mathrm{~V}$ \\
Star G & $2.23 D$ & $2.23 \mathrm{~V}$ & $1 D$ & $1 \mathrm{~V}$ \\
Star H & $3 D$ & $3 V$ & $1.6 D$ & $1.6 \mathrm{~V}$ \\
Star I & $4 D$ & $4 V$ & $2.14 D$ & $2.14 \mathrm{~V}$ \\
Star J & $2 D$ & $2 \mathrm{~V}$ & $3.67 D$ & $3.67 \mathrm{~V}$ \\
\hline
\end{tabular}




\section{Appendix E: Neo-Newtonian Mechanics}

To go beyond this limit of Newtonian mechanics, it can be appealed to the "Neo-Newtonian Mechanics"

This new theory is based on a "variable inertial mass" instead of a variable time.

With the Neo-Newtonian Mechanics, it can be explained:

- the Lorentz transformation: "How to demonstrate the Lorentz factor: variable time vs. variable inertial mass", Journal of Modern Physics, http://file.scirp.org/Html/7-7502022_54203.htm

- the addition of velocities with a sum always lower than the light celerity: "Velocity addition demonstrated from the conservation of linear momenta, an alternative expression", Journal of Modern Physics, http://file.scirp.org/Html/2-7502196_56126.htm

- the radiation emitted in a synchrotron: "Net force $\mathrm{F}=\gamma^{3}$.m.a at high velocity", Journal of Modern Physics, http://file.scirp.org/Html/5-7502665_66042.htm

- the Mercury perihelion precession: "About the ovoid orbits in general, and perihelion precession of Mercury in particular (2)", Millennium Relativity, http://gsjournal.net/Science-Journals/Research\%20Papers/View/6607

- the gravitational light bending: "Hipparcos did not measure directly the light bending!", the General Science Journal, http://gsjournal.net/Science-Journals/Research\%20Papers/View/6998

- the missing of gravitational waves: "Gravitational waves or particles radiation?", Physics Essays, https://www.physicsessays.org/browse-journal-2/product/1588-12-olivier-ser ret-gravitational-waves-or-particle-radiation.html

- the missing of dark matter: "The flat rotation curve of our Galaxy explained within Newtonian mechanics", Physics Essays, https://physicsessays.org/browse-journal-2/product/1240-7-olivier-serret-the -flat-rotation-curve-of-our-galaxy-explained-within-newtonian-mechanics.ht $\underline{\mathrm{ml}}$

- the missing of dark energy: present article

And let us add that based on a stable time whatever the motion of the frame is, the Neo-Newtonian Mechanics is in coherence with the Standard Model of Quantum Mechanics. 